\title{
Pharmacokinetic characterization of favipiravir in patients with COVID-19 and patient outcome
}

\author{
Rezzan Gülhan ${ }^{1}$, Emel Eryüksel ${ }^{1}$, Medine Gülçebi Idriz Oglu ${ }^{1}$, Yekta Çulpan ${ }^{1}$, Aylin \\ Toplu $^{1}$, Derya Kocakaya ${ }^{1}$, Elif Tükenmez Tigen ${ }^{1}$, Buket Ertürk Şengel ${ }^{1}$, Uluhan Sili ${ }^{1}$, \\ Sehnaz Olgun Yildizeli ${ }^{1}$, Baran Balcan ${ }^{1}$, Abdullah Elçi ${ }^{2}$, Cenk Bulut ${ }^{2}$, Atila Karaalp ${ }^{1}$, \\ Hasan Yananli ${ }^{1}$, Abdullah Güner ${ }^{3}$, Mustafa Hatipoglu ${ }^{3}$, Sait Karakurt ${ }^{1}$, Volkan Korten ${ }^{1}$, \\ Neville Ratnaraj ${ }^{4}$, Philip Patsalos ${ }^{4}$, Pinar $\mathrm{Ay}^{1}$, and Filiz Yilmaz Onat ${ }^{1}$ \\ ${ }^{1}$ Marmara University School of Medicine \\ ${ }^{2}$ Istanbul Provincial Health Directorate \\ ${ }^{3}$ Istanbul Health Directorate \\ ${ }^{4}$ University College London Queen Square Institute of Neurology
}

July 2, 2021

\begin{abstract}
Favipiravir is one of the repurposed antiviral medications for the treatment of SARS-CoV-2 infection. Since the dosing regimen is a prominent factor for the success of the antiviral therapy, this prospective observational study aimed to characterize the pharmacokinetic characteristics of favipiravir in COVID-19 patients. Adult patients $(n=21)$ hospitalized for mild to moderate COVID-19 with a positive RT-PCR test, and assigned for favipiravir treatment were included. Favipiravir was administered for 5 days, with a loading dose of $3200 \mathrm{mg}$ and a $1200 \mathrm{mg}$ /day maintenance dose. Serial blood samples were collected on Day-2 and Day-4 of the therapy. Laboratory findings of the patients and in-hospital mortality were assessed. Favipiravir concentrations exhibited high variations and a significant decrease during the treatment of COVID-19. The median favipiravir trough concentration (C0-trough) on Day-2 was $21.26 \mu \mathrm{g} / \mathrm{mL}$ whereas it decreased significantly to $1.61 \mu \mathrm{g} / \mathrm{mL}$ on Day-4, the area under the concentration versus time curve decreased from $345.6 \mu \mathrm{g} . \mathrm{h} / \mathrm{mL}$ to $108.6 \mu \mathrm{g} . \mathrm{h} / \mathrm{mL}$, respectively. Gender seems significant to affect favipiravir concentrations. Day-2-C0-trough of female patients was significantly higher than male patients. Of the 5 patients that died, 4 were male with a significant increase in ferritin levels from Day-0 to Day- 5 compared to surviving patients. In addition, there was a significant decrease in D-dimer and CRP levels in the surviving patients. Our findings indicate that favipiravir concentrations show significant changes during the treatment of COVID-19. Therapeutic drug monitoring may best guide dose adjustments in patients that do not respond to treatment with favipiravir.
\end{abstract}

\section{Hosted file}

Gulhan et al- Pharmacokinetics of favipiravir.docx available at https://authorea.com/users/ 420000/articles/528645-pharmacokinetic-characterization-of-favipiravir-in-patients-withcovid-19-and-patient-outcome 